\title{
Supervised versus unsupervised intake of six-dose artemether-lumefantrine for treatment of acute, uncomplicated Plasmodium falciparum malaria in Mbarara, Uganda: a randomised trial
}

Patrice Piola, Carole Fogg, Francis Bajunirwe, Samuel Biraro, Francesco Grandesso, Eugene Ruzagira, Joseph Babigumira, Isaac Kigozi, James Kiguli, Juliet Kyomuhendo, Laurent Ferradini, Walter Taylor, Francesco Checchi, Jean-Paul Guthmann

\section{Summary}

Background The six-dose regimen of artemether-lumefantrine is effective and is among combination therapies prioritised to replace antimalarials that no longer work in Africa. However, its effectiveness has not been assessed in the field, and could be compromised by poor adherence, incorrect timing of doses, and insufficient intake of fatty foods with every dose. Our aim, therefore, was to assess the effectiveness of artemether-lumefantrine prescribed under routine outpatient conditions, compared with its efficacy when given under supervision to inpatients with acute uncomplicated falciparum malaria.

Methods We did a randomised trial to compare the efficacy, safety, and pharmacokinetics of artemetherlumefantrine when given in a supervised (all doses observed with fatty-food intake; $n=313$ ) or unsupervised (first dose supervised followed by outpatient treatment with nutritional advice; $n=644$ ) setting to patients of all ages (weight $>10 \mathrm{~kg}$ ) with acute, uncomplicated falciparum malaria in Mbarara, Uganda. Our primary endpoint was 28-day, PCR-adjusted, parasitological cure rate. Analysis was by intention to treat and evaluability analysis.

Findings 38 patients were lost to follow-up and one withdrew consent. Day-28 cure rates were $97.7 \%$ (296 of 303) and $\mathbf{9 8 . 0 \%}$ (603 of 615) in the supervised and unsupervised groups, respectively. We recorded 15 non-severe, drug-related adverse events, all of which resolved.

Interpretation Artemether-lumefantrine has a high cure rate irrespective of whether given under supervision with food or under conditions of routine clinic practice. If used as first-line treatment, artemether-lumefantrine could make a substantial contribution to malaria control in Africa, though cost is an issue.

\section{Introduction}

With an estimated 500 million individuals affected every year, malaria is a leading cause of morbidity and mortality in sub-Saharan Africa along with HIV/AIDS. ${ }^{1}$ Of the 1 million deaths caused by malaria worldwide, about $90 \%$ occur in African children, a situation compounded by the emergence of drug resistance. ${ }^{2}$

In Uganda, which had a population of $24 \cdot 7$ million in 2003, an estimated 9.8 million individuals are infected with malaria every year (John Bosco Rwakimari, Ugandan Ministry of Health, Uganda, personal communication; http://www.health.go.ug). To tackle malaria-related mortality and morbidity, the Ugandan Ministry of Health $(\mathrm{MoH})$ is concentrating on early diagnosis and effective treatment of the disease. In the 1970 s and the 1980s, high malaria awareness in the population and easy access to cheap and effective antimalarials such as chloroquine and sulfadoxinepyrimethamine ensured the disease was reasonably well controlled. However, resistance to these drugs is now widespread in Uganda and in other parts of east Africa, with adverse consequences for malaria control. ${ }^{3-7}$

The $\mathrm{MoH}$-recommended first-line antimalarial drug in Uganda is chloroquine combined with sulfadoxine- pyrimethamine, ${ }^{6}$ though introduction of artemisininbased combination treatments (ACTs) is planned for 2005. ${ }^{8}$ Day-28 cure rates of $52 \%,{ }^{9} 65 \%,{ }^{7}$ and $77 \%{ }^{10}$ have been reported for this combination in different parts of Uganda. ACTs are judged effective in Africa, where they improve cure rates and reduce gametocyte carriage compared with presently used monotherapies. ${ }^{11,12}$ To combat drug-resistant malaria in Africa, WHO advocates the adoption of ACTs as first-line treatment. ${ }^{2}$ The use of the non-ACT combination of amodiaquine and sulfadoxine-pyrimethamine is considered by some as an interim measure while waiting for ACTs to become widely available. Day-28 efficacy rates of this combination were $84 \%$ and $90 \%$ in two studies in Uganda. ${ }^{79}$

Artemether-lumefantrine (Coartem, Novartis Pharma, Basel, Switzerland) is the only fixed-dose formulation ACT on the WHO essential drug list. However, the combination is not registered for use in pregnant women, and was not registered for children under $10 \mathrm{~kg}$ in weight when we did our trial. Results of studies ${ }^{13-15}$ from southeast Asia show that the six-dose regimen of artemether-lumefantrine has cure rates of more than $96 \%$, is well tolerated, and has a good safety profile when
Lancet 2005; 365: 1467-73

See Comment page 1441

Epicentre, 8 rue Saint-Sabin, 75011 Paris, France (P Piola MD, C Fogg MSc, S Biraro MPH, F Grandesso MSc, E Ruzagira MBBS, J Babigumira MBBS, I Kigozi MBBS, J Kyomuhendo, L Ferradini PhD, F Checchi MHS, J-P Guthmann PhD); Mbarara University of Science and Technology, Mbarara, Uganda (F Bajunirwe MBBS, J Kiguli); and WHO/Special Programme for Research and Training in Tropical Diseases (TDR), Geneva, Switzerland (W Taylor MRCP)

Correspondence to: Dr Patrice Piola uganda@epicentre.msf.org 
given under supervision. However, a study ${ }^{16}$ of the fourdose regimen done in Thailand resulted in a cure rate of only $83 \%$. There are limited data on use of the six-dose regimen in Africa, though supervised administration of the combination had a $99 \%$ cure rate when assessed at day 14 in Burundian children. ${ }^{17}$ Day-14 efficacy of the four-dose regimen was good in the Gambia (93\%), ${ }^{18}$ but modest in Tanzania $(86 \%){ }^{19}$

Several factors could reduce considerably the effectiveness of artemether-lumefantrine under field conditions in Africa. Adherence to the complicated, twice-daily, three-day regimen might be suboptimum. To increase lumefantrine absorption, all doses should be correctly spaced and taken with food. The manufacturer recommends an interval of $8 \mathrm{~h}$ between the first and the second dose, $24 \mathrm{~h}$ between the first and the third dose, and 12 hourly intervals between doses thereafter. Lumefantrine absorption is greatly increased when taken with food, especially fatty foods. Plasma concentrations of the drug are low after initial doses, when patients are typically anorexic; levels increase in parallel with improved appetite..$^{20,21}$ The day-7 plasma concentration of lumefantrine is a predictor of therapeutic response. Concentrations of less than $280 \mu \mathrm{g} / \mathrm{L}$ were associated with an increased risk of treatment failure in Thailand. ${ }^{22}$ This concentration is judged to be the in-vivo minimum inhibitory concentration for multidrug-resistant falciparum parasites. ${ }^{23}$ Artemether-lumefantrine is expensive, with the WHO negotiated price ranging from US $\$ 0.9$ in the smallest children to $\$ 2.40$ for an adult course. This high cost could deter African Ministries of Health from recommending artemether-lumefantrine in the absence of donor funds.

In Mbarara, southwestern Uganda, the efficacies of chloroquine and sulfadoxine-pyrimethamine are low. ${ }^{24}$ Furthermore, 3 days of treatment with artesunate plus sulfadoxine-pyrimethamine yield a day-28, PCRcorrected cure rate of only $74 \% .{ }^{25}$ A new treatment option is needed. Our aim, therefore, was to assess the effectiveness of artemether-lumefantrine prescribed under routine outpatient conditions, compared with the efficacy of the combination given under supervision to inpatients with acute uncomplicated falciparum malaria.

\section{Methods \\ Participants}

Between December, 2002, and January, 2004, we did an open-label, randomised trial at the Mbarara University Teaching Hospital, which is the regional referral hospital for the western region of Uganda. Mbarara town (population of about 69000 in 2002) is in western Uganda, an area of perennial malaria transmission where the disease has two seasonal peaks that coincide with the rainy seasons (March-June and SeptemberDecember). Plasmodium falciparum is the predominant species (95\%, unpublished data) and is resistant to chloroquine and sulfadoxine-pyrimethamine. ${ }^{24}$
Potential participants were referred from the hospital outpatient clinic and local municipality dispensaries to the Epicentre clinic. Our inclusion criteria were: fever in the previous $24 \mathrm{~h}$ or confirmed fever (axillary temperature $>37 \cdot 5^{\circ} \mathrm{C}$ ); weight of $10 \mathrm{~kg}$ or more; monoinfection with $P$ falciparum, parasitaemia of 500-100 000 trophozoites $/ \mu \mathrm{L}$, no danger signs (unable to drink or feed, repeated vomiting, convulsions during the present illness, lethargic or unconscious state, unable to sit or stand), and no signs of severe malaria; ${ }^{26}$ no other clinically significant illnesses; not pregnant (confirmed by history and a negative urinary pregnancy test); and residence in Mbarara Municipality.

All patients or their guardians provided written informed consent. We wrote the study protocol following the principles of the Declaration of Helsinki, and it was approved by three ethics committees: the Mbarara Faculty of Medicine, the Mbarara University of Science and Technology, and the Uganda National Committee for Science and Technology.

\section{Procedures}

We stratified arbitrarily the study population so as to compare the efficacy of supervised and unsupervised treatment in three age groups: children younger than age 5 years; children aged 5-14 years; and patients aged 15 years or older. We used these age groups as surrogate markers of malaria-acquired immunity and possible adherence patterns.

After inclusion, we randomly assigned participants to receive either supervised or unsupervised artemetherlumefantrine, according to a computer-generated randomisation list designed in blocks of five. We used a one-to-one, a one-to-two, and a one-to-four (supervisedto-unsupervised) randomisation schedule for children aged younger than 5 years, those aged 5-14 years, and for patients aged 15 years or older, respectively. We randomly assigned patients in an unequal way in the different age groups to reduce to a minimum the time that patients would be away from school or work.

Treatment allocation was concealed. Every inclusion number corresponded to a sealed envelope, containing a card with the treatment allocation ("supervised" or "unsupervised") written on it. We prescribed artemetherlumefantrine in its blister pack. Tablets contained $20 \mathrm{mg}$ of artemether and $120 \mathrm{mg}$ of lumefantrine. The packs have in them pictures to show how tablets should be given, and contain two blisters for every day, containing one, two, three, or four tablets dependent on the weight group. The regimen consisted of one $(10-14.9 \mathrm{~kg})$, two (15-24.9 kg), three (25-34.9 kg), or four (>35 kg) tablets twice daily for 3 days. Tablets were crushed in water for young children unable to swallow them whole.

We admitted patients assigned to the supervised group to an observation ward for the duration of treatment. All doses were administered by a study nurse, and a fatty meal composed of about $300 \mathrm{~mL}$ of milk (10 g fat) and 
$30 \mathrm{~g}$ of peanuts (13 $\mathrm{g}$ fat) was given either before or just after drug administration. If the patient with malaria was being breastfed, mothers were encouraged to continue nursing. We administered doses at the times recommended by the manufacturer: $0 \mathrm{~h}, 8 \mathrm{~h}, 24 \mathrm{~h}, 36 \mathrm{~h}$, $48 \mathrm{~h}$, and $60 \mathrm{~h}$. We repeated doses if patients vomited within $30 \mathrm{~min}$ of administration. Any prescribed concomitant drugs-eg, paracetamol-were administered and recorded. We gave patients in the unsupervised group their first dose at the clinic, observed them for vomiting, and then discharged them with their blister pack. We advised patients or their parents to combine the treatment with fatty meals or breastmilk. We emphasised the need to administer the second dose exactly $8 \mathrm{~h}$ after the first supervised dose.

We followed up patients for 28 days, according to a schedule of visits on days $3,7,14,21$, and 28 for clinical assessments and laboratory tests. We also saw and assessed the supervised patients daily for the first 3 days. At every visit, we prepared and read 3\% Giemsa stained thick and thin smears to establish species (thin smear), quantify asexual parasitaemia (per $\mu \mathrm{L}$ ), and detect gametocytaemia (number per 1000 white cells on a thick smear), according to WHO standard methods. We judged a slide negative after examination of 200 highpower fields. We collected capillary blood on Isocode kits (Schleicher and Schuell, Dassel, Germany) before treatment (day 0) and on the day of recurrent parasitaemia (in case this arose after day 3) for PCR genotyping. We distinguished reinfections from recrudescences by comparing the pretreatment and posttreatment genotypes of the $P$ falciparum gene loci coding for the merozoite surface proteins 1 and 2 (MSP-1 and MSP-2) and the glutamate rich protein (GLURP). ${ }^{27} \mathrm{We}$ measured concentration of haemoglobin (capillary blood) with a Hemocue B-Hemoglobin apparatus (Hemocue, Angelholm, Sweden) on days 0 and 28.

We encouraged patients and their guardians to return to the clinic at any time if their health or that of their children deteriorated. Individuals who did not return for scheduled follow-up visits were traced the same day. Patients for whom artemether-lumefantrine was ineffective or who vomited persistently after treatment received rescue treatment with quinine hydrochloride (10 mg per kg every $8 \mathrm{~h}$ for 7 days).

Our primary endpoint was cure at 28 days. We considered the reasons some patients were not cured as either: parasitological in the case of PCR-confirmed recrudescent parasitaemia after day 3 (we recorded patients with indeterminate or missing PCR results as not having been cured, and patients reinfected as cured); or non-parasitological if the patient had taken any drugs with antimalarial activity during follow-up (other than rescue treatment), if treatment with artemether-lumefantrine had been interrupted because of an adverse event, or if a serious adverse event during follow-up arose, irrespective of whether there was an association with the study drug.
Our secondary endpoints were the proportion of afebrile patients on days 1,2 , and 3, post-treatment gametocyte carriage, and haematological recovery.

To explore a possible relation between lumefantrine concentrations and treatment failure, we took whole venous blood $(4 \mathrm{~mL})$ on days 3 and 7 from 70 patients in every treatment and age group. Patients were selected by simple random sampling with a computer-generated random-sampling list. We froze the serum at $-80^{\circ} \mathrm{C}$ before shipping it to the Bioanalytics and Pharmacokinetics department of Novartis Pharma (Rueil Malmaison, France). Lumefantrine concentrations were ascertained by high performance liquid chromatography, according to a previously published method (minimum detectable lumefantrine concentration of $5 \mu \mathrm{g} / \mathrm{L}$, within-day and dayto-day coefficients of variation of $1 \cdot 8-4 \cdot 0$ and $1 \cdot 8-4 \cdot 2 \%$, respectively),$^{28}$ and blinded to patients' endpoints.

During follow-up, we assessed patients clinically for the presence of adverse events (or any untoward medical occurrence from day 0 to day 28 in a participant who took at least one dose of artemether-lumefantrine) and serious adverse events, defined as an adverse event that was fatal, life threatening, permanently disabling, led to admission to hospital for treatment, or caused a congenital abnormality. We grouped adverse events and graded them by intensity with the Common Toxic Criteria (National Cancer Institute), before ordering them into six drug-event relation categories: none, unlikely, possible, probable, definite, and unknown. We monitored all patients with adverse events until their health improved.

\section{Statistical analysis}

We assessed our primary endpoint and all secondary endpoints by a modified intention-to-treat analysis in which participants without an ascertained study outcome (because of missed study visit or withdrawal of consent) were excluded. By way of comparison, we also did an evaluability analysis. In the evaluability analysis of the primary endpoint, we also excluded patients who were not cured for reasons other than parasitological factors, and for whom PCR data were missing or reinfection arose. We also excluded from the evaluability analysis patients who did not meet all of our inclusion criteria, were randomised to the wrong group, did not take all doses correctly (supervised group only), had nonfalciparum parasitaemia during follow-up, or received unwarranted rescue treatment.

Based on limited published work, we assumed a day-28 cure rate in the supervised group of $96 \%$ among those younger than age 5 years, and of $97 \%$ among older patients. For the unsupervised group we assumed a cure rate of $85 \%$ in all age groups. We calculated the sample size to show an $11 \%$ and a $12 \%$ difference in cure rates for children aged younger than 5 years and for older age groups, respectively. We assumed a type 1 error of $5 \%$, a power of $80 \%$, and a $10 \%$ drop-out rate. As such, the required sample sizes were (supervised-to- 


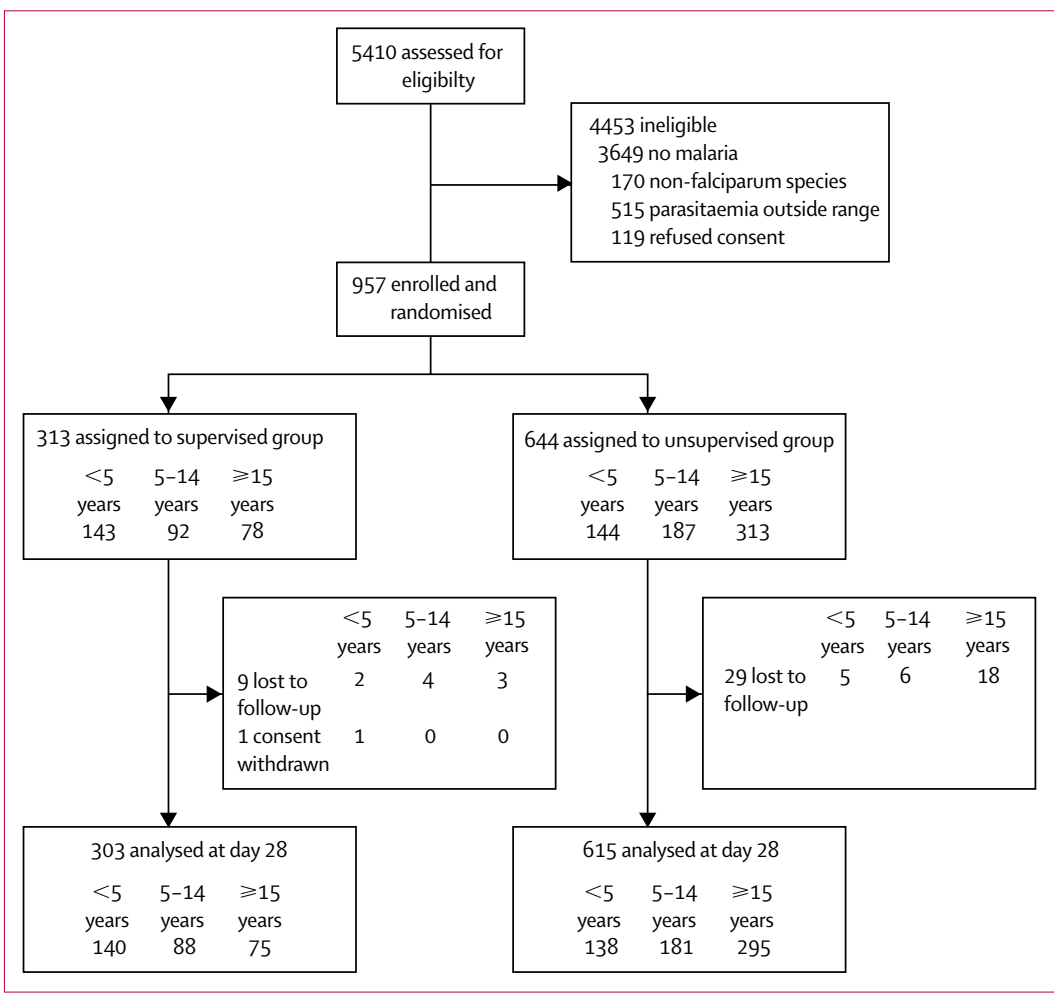

Figure: Trial profile (intention-to-treat analysis) unsupervised): 141-to-141 $\quad(<5 \quad$ years $), \quad$ 91-to-183 (5-14 years), and 78-to-312 ( $\geqslant 15$ years).

We double-entered data with EpiData 3.0 software (EpiData Association, Odense, Denmark), and cleaned and analysed the data with Stata 8.2 (StataCorp, College Station, Texas, USA). We compared categorical variables with a $\chi^{2}$ test. To compare continuous data between groups, we used the Mann-Whitney $U$ test for the day 0 asexual parasite counts and lumefantrine concentrations (not normally distributed by Kurtosis test) and the unpaired $t$ test for age, weight, temperature, and haemoglobin concentrations.

\section{Role of the funding source}

Médecins Sans Frontières (MSF) funded the project and participated in protocol development, but had no subsequent role in undertaking the study or in this publication. Novartis Pharma funded and did the pharmacokinetics analysis, but had no role in any other trial-related activity or this publication, and had no access to study data.

\section{Results}

The figure shows the trial profile. We screened 5410 patients for eligibility, of whom 957 (18\%) were enrolled. Baseline characteristics were comparable across groups (table 1), with the exception of asexual

\begin{tabular}{|c|c|c|c|c|c|c|}
\hline & \multicolumn{2}{|l|}{$<5$ years } & \multicolumn{2}{|l|}{$5-14$ years } & \multicolumn{2}{|l|}{$\geqslant 15$ years } \\
\hline & Supervised $(n=143)$ & Unsupervised $(n=144)$ & Supervised ( $n=92)$ & Unsupervised $(\mathrm{n}=187$ ) & Supervised ( $\mathrm{n}=78$ ) & Unsupervised $(n=313)$ \\
\hline Age (years) & $2 \cdot 8(1.5)$ & $2 \cdot 7(1.0)$ & $8.5(2.8)$ & $8 \cdot 3(2 \cdot 8)$ & $27 \cdot 7(10 \cdot 4)$ & $28 \cdot 5(11 \cdot 1)$ \\
\hline Male (number [\%]) & $83(58 \%)$ & $80(56 \%)$ & $44(48 \%)$ & $86(46 \%)$ & $35(45 \%)$ & $126(40 \%)$ \\
\hline Female (number [\%]) & $60(42 \%)$ & $64(44 \%)$ & $48(52 \%)$ & $101(54 \%)$ & $43(55 \%)$ & $187(60 \%)$ \\
\hline Weight (kg) & $13 \cdot 2(3 \cdot 4)$ & $13.0(2 \cdot 5)$ & $24 \cdot 4(7 \cdot 2)$ & $25 \cdot 0(7 \cdot 9)$ & $56 \cdot 3(9 \cdot 1)$ & $56 \cdot 5(9 \cdot 3)$ \\
\hline Haemoglobin (g/dL) & $9 \cdot 6(1.9)$ & $9 \cdot 5(1.9)$ & $11 \cdot 2(1 \cdot 7)$ & $11 \cdot 4(1 \cdot 6)$ & $13 \cdot 2(1 \cdot 9)$ & $12 \cdot 9(2 \cdot 0)$ \\
\hline Temperature $\left({ }^{\circ} \mathrm{C}\right)$ & $37 \cdot 4(1 \cdot 2)$ & $37 \cdot 5(1 \cdot 2)$ & $37 \cdot 3(1 \cdot 1)$ & $37 \cdot 0(1 \cdot 1)$ & $36 \cdot 7(1 \cdot 0)$ & $36 \cdot 6(1 \cdot 0)$ \\
\hline Median (range) parasite density per $\mu \mathrm{L}$ & $11421(125-140278)$ & $18551(350-130521)$ & $15668(676-94205)$ & $10017(597-128264)$ & $6250(662-115214)$ & 5909 (394-97 657) \\
\hline Splenomegaly (number [\%]) & $49(34 \%)$ & $29(20 \%)$ & $27(29 \%)$ & $51(27 \%)$ & $10(13 \%)$ & $30(10 \%)$ \\
\hline Hepatomegaly (number [\%]) & $8(6 \%)$ & $1(1 \%)$ & $4(4 \%)$ & $4(2 \%)$ & 0 & $8(3 \%)$ \\
\hline Gametocyte carriage (number [\%]) & $19(13 \%)$ & $17(12 \%)$ & $3(3 \%)$ & $14(8 \%)$ & $1(1 \%)$ & $18(6 \%)$ \\
\hline
\end{tabular}

Table 1: Baseline characteristics

\begin{tabular}{|c|c|c|c|c|c|c|c|c|}
\hline & \multicolumn{4}{|l|}{ Supervised } & \multicolumn{4}{|l|}{ Unsupervised } \\
\hline & $<5$ years & $5-14$ years & $\geqslant 15$ years & Total & $<5$ years & $5-14$ years & $\geqslant 15$ years & Total \\
\hline No cure & 5 & 2 & 0 & 7 & 4 & 6 & 2 & 12 \\
\hline Parasitological recrudescence & 0 & 0 & 0 & 0 & 0 & 0 & 0 & 0 \\
\hline Recurrent parasites, no $\mathrm{PCR}$ result & 1 & 1 & 0 & 2 & 1 & 2 & 1 & 4 \\
\hline Other antimalarial intake & 2 & 1 & 0 & 3 & 2 & 3 & 1 & 6 \\
\hline AL interrupted by severe adverse event & 2 & 0 & 0 & 2 & 1 & 1 & 0 & 2 \\
\hline Cure & 135 & 86 & 75 & 296 & 135 & 175 & 293 & 603 \\
\hline Absence of parasites after day 3 & 130 & 85 & 74 & 289 & 122 & 169 & 291 & 582 \\
\hline Reinfection detected by PCR & 5 & 1 & 1 & 7 & 13 & 6 & 2 & 21 \\
\hline Cure rate $(\%, 95 \% \mathrm{Cl})$ & $\begin{array}{l}135 \text { of } 140 \\
(96 \cdot 4 \%, 91 \cdot 4-98 \cdot 7)\end{array}$ & $\begin{array}{l}86 \text { of } 88 \\
(97 \cdot 7 \%, 91 \cdot 2-99 \cdot 6)\end{array}$ & $\begin{array}{c}75 \text { of } 75 \\
(100 \cdot 0 \%, 93 \cdot 9-100)\end{array}$ & $\begin{array}{l}296 \text { of } 303 \\
(97 \cdot 7 \%, 95 \cdot 1-99 \cdot 0)\end{array}$ & $\begin{array}{l}135 \text { of } 139 \\
(97 \cdot 1 \%, 92 \cdot 3-99\end{array}$ & $\begin{array}{l}175 \text { of } 181 \\
\text { 1) }(96 \cdot 7 \%, 92 \cdot 6-98 \cdot 6)\end{array}$ & $\begin{array}{l}293 \text { of } 296 \\
(99.0 \%, 96.8-99.7)\end{array}$ & $\begin{array}{l}603 \text { of } 615 \\
(98 \cdot 0 \%, 96 \cdot 5-98 \cdot 9)\end{array}$ \\
\hline $\mathrm{AL}=$ artemether-lumefantrine. & & & & & & & & \\
\hline
\end{tabular}




\begin{tabular}{|c|c|c|c|c|c|c|}
\hline & \multicolumn{2}{|l|}{$<5$ years } & \multicolumn{2}{|l|}{$5-14$ years } & \multicolumn{2}{|l|}{$\geqslant 15$ years } \\
\hline & Supervised & Unsupervised & Supervised & Unsupervised & Supervised & Unsupervised \\
\hline \multicolumn{7}{|l|}{ Day 3} \\
\hline Number of patients & 69 & 58 & 85 & 83 & 74 & 73 \\
\hline Mean $(S D)$ concentration $(\mu \mathrm{g} / \mathrm{L})$ & $7050(3560)$ & $4064(3609)$ & $6906(3683)$ & $4389(3155)$ & $5596(2691)$ & $4090(3080)$ \\
\hline \multicolumn{7}{|l|}{ Day 7} \\
\hline Number of patients & 68 & 59 & 85 & 83 & 74 & 73 \\
\hline Mean $(S D)$ concentration $(\mu \mathrm{g} / \mathrm{L})$ & $376(217)$ & $249(245)$ & $526(556)$ & $307(208)$ & $460(288)$ & $317(190)$ \\
\hline Number (\%) below 280 g/L & $25(37 \%)$ & $42(71 \%)$ & $24(28 \%)$ & $47(57 \%)$ & $18(24 \%)$ & $33(45 \%)$ \\
\hline
\end{tabular}

parasitaemia in children younger than age 5 years. Day 0 gametocytaemia declined with increasing age. During follow-up, we withdrew 69 patients (7\%) from the evaluability analysis, of whom: 19 did not meet all inclusion criteria, three were randomised in error, four interrupted treatment, seven had recurrent nonfalciparum parasitaemia, nine took other antimalarials, 24 had a reinfection, and three had an indeterminate PCR result. 38 patients (4\%) defaulted (two of these were among those withdrawn from the evaluability group) and one withdrew consent, leaving a sample of 918 (96\%) for the intention-to-treat analysis and 851 (89\%) for the evaluability analysis.

Cure rates were high with both forms of analysis. In the intention-to-treat analysis, the overall cure rates were $97.7 \%$ in the supervised group and $98.0 \%$ in the unsupervised group (table 2). In the evaluability analysis, the cure rate was $100 \%$ in both groups (95\% CI 98-100 and 99-100 for supervised and unsupervised, respectively). None of the differences between treatment groups and age groups were significant $(p>0 \cdot 20$ for all comparisons).

Of the 918 patients included in the intention-to-treat analysis, 296 (32\%) had a fever on day 0. In the supervised group, $79 \%$ (92 of 116) and 100\% (116 of 116) were afebrile on days 1 and 3 , respectively. In the unsupervised group, these proportions were $97 \%$ (175 of 180) on day 3 and $100 \%$ on day 7 . The proportion of patients with gametocytes declined from: 8\% (69 of 918) on day 0 to $2 \%$ (17 of 898 ) on day 7 for the entire cohort; from $16 \%$ (35 of 279 ) to $4 \%$ (12 of 273 ) in children younger than age 5 years; from $6 \%$ (16 of 269) to $1 \%$ (two of 263) in children aged 5-14 years; and from 5\% (18 of 370 ) to $1 \%$ (three of 362) in individuals aged 15 years or older. Only three $(0 \cdot 3 \%)$ patients $(\geqslant 15$ years) were still gametocytaemic on day 14 (two unsupervised, one supervised) and only one had gametocytes after day 14 . By day 28, mean haemoglobin concentrations had increased in all age groups with no significant difference between the supervised and unsupervised patients ( $t$ tests for $<5$ years: $p=0 \cdot 53$; for $5-14$ years: $p=0 \cdot 98$; and for $\geqslant 15$ years: $p=0 \cdot 91$ ). The mean (SD) fractional change was significantly higher in the two younger age groups compared with the adults $(\mathrm{p}<0 \cdot 0001)$ : $2 \cdot 1(1 \cdot 70) \mathrm{g} / \mathrm{dL}(<5$ years $), 1 \cdot 3(1 \cdot 64) \mathrm{g} / \mathrm{dL}(5-14$ years $)$, and $0 \cdot 3(1.58) \mathrm{g} / \mathrm{dL}$ ( $\geqslant 15$ years).
We measured blood concentrations of lumefantrine in 228 supervised patients and in 214 unsupervised individuals. The mean lumefantrine concentrations $(\mu \mathrm{g} / \mathrm{L})$ were significantly lower $(\mathrm{p}<0 \cdot 0001)$ on days 3 (4210 vs 6512) and 7 (319 vs 461) in the unsupervised versus supervised group; this pattern was seen for every age group (table 3 ). On day 7 , all age groups had a significantly higher proportion of patients with lumefantrine concentrations of less than $280 \mu \mathrm{g} / \mathrm{L}$ in the unsupervised group than in the supervised group (table 3). Detailed findings on predictors of lumefantrine concentration, and on the effect on varying weightadjusted lumefantrine doses on both lumefantrine and reinfection risk will be presented elsewhere.

We mistakenly included four pregnant women in the study and treated them with full courses of artemetherlumefantrine during the first trimester. They were followed up closely and all had normal deliveries. All newborn babies were healthy and subsequent follow-up for 1 year has not revealed any developmental delays. Overall, there were 521 reported adverse events, the most common of which were cough (12\%), upper respiratory tract infection $(11 \%)$, abdominal pain $(7 \%)$, headache $(6 \%)$, fever $(4 \%)$, diarrhoea $(3 \%)$, rash $(3 \%)$, dizziness $(3 \%)$, anorexia $(2 \%)$, and vomiting $(2 \%)$. The breakdown of drug-event associations was: three $(0.6 \%)$ definite, $12(2 \%)$ probable, 33 (6\%) possible, 360 (69\%) unlikely, and $113(22 \%)$ not related to artemether-lumefantrine. There were no differences between the groups (data not shown). All of the 15 definitely or probably drug-related adverse events were of mild or moderate severity, and resolved: three vomiting $(0$ mild $/ 3$ moderate), three diarrhoea $(1 / 2)$, three anorexia $(2 / 1)$, two abdominal pain $(0 / 2)$, two palpitations $(0 / 2)$, one nausea $(0 / 1)$, and one pruritus $(0 / 1)$. There were eight severe adverse events, which were all malaria-related or intercurrent illnesses unrelated to the study drug: convulsions 5 min after first drug intake, severe malaria on day 0 , measles, seizure with febrile semiconsciousness, ectopic pregnancy, pelvic inflammatory disease, obstructed inguinal hernia, and death due to pneumonia in an HIV-positive man.

\section{Discussion}

Our results indicate that artemether-lumefantrine has a high cure rate-exceeding 96\%-irrespective of whether given under supervision with food or under conditions of 
routine clinic practice. This finding confirms the efficacy of the six-dose regimen reported by investigators in southeast Asia and Burundi. ${ }^{13-15,17}$ Our results are also consistent with an earlier study done at the same clinic as this one and showing $90 \%$ adherence to outpatient treatment with artemether-lumefantrine. ${ }^{29}$ Provision of a short explanation about how to take a drug to patients at the point of prescription is, seemingly, a simple but important intervention to enhance adherence. Although this research clinic might over-rate population adherence to artemether-lumefantrine, our encouraging data should facilitate a decision on whether to introduce this drug as first-line treatment for P falciparum malaria in Uganda.

Our underlying hypothesis was that a trial of artemether-lumefantrine efficacy alone, done under ideal conditions of drug and food administration, was likely to yield an overly optimistic estimate of the actual cure rate and ignore the possible negative effect of factors such as poor lumefantrine absorption under normal conditions of food intake, poor adherence to the obligatory 8-h delay between the first and the second dose, and early interruption of treatment because of rapid symptomatic relief. Nevertheless our findings suggest that artemether-lumefantrine would be highly effective in an African clinic provided that clear explanations on how to take the drug are given. It is noteworthy, however, that no matter how closely we have tried to mimic the real-life situation, study procedures always impose an artificial environment that indirectly promotes adherence, and therefore overestimates the true drug efficacy in the unsupervised group.

Artemether-lumefantrine had a profound effect on gametocyte carriage; only one patient had gametocytes after day 14. This factor might confer additional benefit to the community by reducing transmission of the disease if the drug were widely deployed in our setting. Other ACTs also inhibit gametocyte development. ${ }^{11}$ By contrast, sulfadoxine-pyrimethamine monotherapy is associated with high gametocyte carriage rates, a major disadvantage of its use. ${ }^{25}$ As with other efficacious antimalarials, there was good haematological recovery especially in the youngest and most vulnerable age group. Artemetherlumefantrine was well tolerated and there were no druginduced serious adverse events, which is consistent with other trials. ${ }^{30}$ Additionally, four babies were born to pregnant women who had been exposed inadvertently to artemether-lumefantrine in the first trimester of pregnancy. They showed no abnormality at birth and are well after a year of monthly follow-up visits.

We measured blood lumefantrine concentrations on days 3 and 7. A day-7 concentration of less than $280 \mu \mathrm{g} / \mathrm{L}$ has been used as a pharmacokinetic marker of treatment failure in western Thailand, an area of highly drugresistant $P$ falciparum. ${ }^{23,31}$ Although day-7 lumefantrine concentrations were significantly lower and more frequently less than $280 \mu \mathrm{g} / \mathrm{L}$ in the unsupervised group, a fairly high proportion of the supervised patients also had concentrations below this marker. These data indicate that supervised drug administration results in improved lumefantrine concentrations, but does not guarantee high day-7 concentrations. However, in this area of Uganda, low day-7 lumefantrine concentrations did not affect the day-28 cure rate. Our fairly short follow up could nonetheless have missed late recrudescent infections, and extending the follow up of artemether-lumefantrine to 42 days is recommended. ${ }^{32}$ Malaria-acquired immunity is a probable contributing factor in our high cure rates, but another important issue is the intrinsic sensitivity of local $P$ falciparum parasites to lumefantrine. Although we did not measure this factor in Mbarara, African isolates have considerably lower IC50 values than those from western Thailand, where the high IC50 values are probably explained by the in-vitro cross-resistance between lumefantrine and mefloquine and halofantrine. ${ }^{3133,34} \mathrm{~A}$ day-7 lumefantrine concentration of less than $280 \mu \mathrm{g} / \mathrm{L}$ as a pharmacokinetic predictor that treatment will not work might not be applicable to all regions, and further research should be done to assess the appropriate threshold as a function of parasite sensitivity.

If, as we believe, parasites in this area are highly sensitive to lumefantrine and artemether, then now is the best time for the Ugandan $\mathrm{MoH}$ to introduce the study combination to reduce the likelihood that resistance will develop..$^{23}$ Every effort should be made to ensure full adherence to this valuable ACT to maximise protection from resistance and realise the possibility of a reduction in malaria transmission. We could not show to what extent high effectiveness was attributable to the improved, sealed-blister design of the packaging of artemether-lumefantrine, consisting of visual depictions of when to take each dose appropriate for non-literate users; nevertheless, our findings do strongly suggest that such a mode of packaging favours high adherence and should be standard for all ACT combinations. In a previous study, ${ }^{29}$ we showed high $(90 \%)$ adherence to artemether-lumefantrine in our clinic setting. One major drawback of the combination remains its cost. Many African countries will not be able to afford artemetherlumefantrine for public-sector use without external support, such as from the Global Fund. This factor could also compromise adherence, since African patients and caregivers often use incomplete doses, keeping the remaining tablets for the next attack of malaria. ${ }^{35}$

The six-dose regimen of artemether-lumefantrine is a promising option as a replacement for antimalarial therapies that no longer work in Uganda and other African countries. Deployment on a wide scale should be undertaken in parallel with research into effectiveness, pharmacovigilance, and resistance monitoring.

Contributors

P Piola was the main investigator. P Piola and J-P Guthmann cowrote the first draft of this paper; all coauthors contributed to the final version. F Checchi designed the study protocol with the support of the Protocol Development Team. C Fogg, F Bajunirwe, S Biraro, F Grandesso,

E Ruzagira, J Babigumira, I Kigozi, J Kiguli, and J Kyomuhendo were in 
charge of trial recruitment and follow-up, and contributed to data analysis. L Ferradini implemented and supervised PCR analysis. W Taylor assisted with protocol design and was the Good Clinical Practice adviser.

\section{Conflict of interest statement}

We declare that we have no conflict of interest. The opinions expressed in this article are not to be construed as representing those of WHO.

\section{Acknowledgments}

We thank the Mbarara health authorities, municipality clinics, staff of the Mbarara University of Science and Technology, and the following trial team members: Stephen Mugisha, Job Baguma, Ismael Sekimuli, Alex Muhumuza, Enid Nuwagaba, Joy Musabe, Winnie Lamulatu, Saidat Nabiyiki, Sulaiman Muwanga, Frederick Mutebi,

Benon Tumwebaze, Bernice Anyango, Jeninah Atwebembeire,

Tinka Kyoshabiire, Hassan Kivumbi, Faith Kabanda, and

Grace Kyomugisha. We also thank the East African Network for

Monitoring Antimalarial Treatment (EANMAT) and the Ugandan Ministry of Health (Malaria Control Programme); Georges Snounou for quality control and advice on PCR analyses; Médecins Sans FrontièresFrance staff for logistical support; and Dominique Legros,

François Nosten, and Piero Olliaro for precious advice on study design.

This study was funded by Médecins Sans Frontières. Novartis Pharma funded the pharmacokinetics analysis, and we thank its staff, including Gilbert Lefevre, for their support.

\section{References}

1 WHO. The Africa malaria report. Geneva: World Health Organization, 2003.

2 WHO. Position of WHO's roll back malaria department on malaria treatment policy. Geneva: World Health Organization, 2003.

3 Campbell CC, Chin W, Collins WE, Teutsch SM, Moss DM. Chloroquine-resistant Plasmodium falciparum from East Africa: cultivation and drug sensitivity of the Tanzanian I/CDC strain from an American tourist. Lancet 1979; 2: 1151-54.

4 Dieckmann A, Jung A. Mechanisms of sulfadoxine resistance in Plasmodium falciparum. Mol Biochem Parasitol 1986; 19: 143-47.

5 Ronn AM, Msangeni HA, Mhina J, Wernsdorfer WH, Bygbjerg IC High level of resistance of Plasmodium falciparum to sulfadoxinepyrimethamine in children in Tanzania. Trans $\mathrm{R}$ Soc Trop Med Hyg 1996; 90: 179-81.

6 Kamya MR, Dorsey G, Gasasira A, et al. The comparative efficacy of chloroquine and sulfadoxine-pyrimethamine for the treatment of uncomplicated falciparum malaria in Kampala, Uganda.

Trans R Soc Trop Med Hyg 2001; 95: 50-55.

7 Staedke SG, Mpimbaza A, Kamya MR, Nzarubara BK, Dorsey G, Rosenthal PJ. Combination treatments for uncomplicated falciparum malaria in Kampala, Uganda: randomised clinical trial. Lancet 2004; 364: 1950-57.

8 WHO. Global AMDP Database: AFRO. http://mosquito.who.int/ amdp/amdp_afro.htm (accessed April 6, 2005).

9 Talisuna AO, Nalunkuma-Kazibwe A, Bakyaita N, et al. Efficacy of sulphadoxine-pyrimethamine alone or combined with amodiaquine or chloroquine for the treatment of uncomplicated falciparum malaria in Ugandan children. Trop Med Int Health 2004; 9: 222-29.

10 Checchi F, Piola P, Kosack C, et al. Antimalarial efficacy of sulfadoxine-pyrimethamine, amodiaquine and a combination of chloroquine plus sulfadoxine-pyrimethamine in Bundi Bugyo, western Uganda. Trop Med Int Health 2004; 9: 445-50.

11 Adjuik M, Babiker A, Garner P, Olliaro P, Taylor W, White N. Artesunate combinations for treatment of malaria: meta-analysis. Lancet 2004; 363: 9-17.

12 Targett G, Drakeley C, Jawara M, et al. Artesunate reduces but does not prevent posttreatment transmission of Plasmodium falciparum to Anopheles gambiae. J Infect Dis 2001; 183: 1254-59.

13 van Vugt M, Angus BJ, Price RN, et al. A case-control auditory evaluation of patients treated with artemisinin derivatives for multidrug-resistant Plasmodium falciparum malaria. Am J Trop Med Hyg 2000; 62: 65-69.

14 van Vugt M, Ezzet F, Nosten F, et al. No evidence of cardiotoxicity during antimalarial treatment with artemether-lumefantrine. Am J Trop Med Hyg 1999; 61: 964-67.

15 Lefevre G, Looareesuwan S, Treeprasertsuk S, et al. A clinical and pharmacokinetic trial of six doses of artemether-lumefantrine for multidrug-resistant Plasmodium falciparum malaria in Thailand. Am J Trop Med Hyg 2001; 64: 247-56.

16 van Vugt M, Brockman A, Gemperli B, et al. Randomized comparison of artemether-benflumetol and artesunate-mefloquine in treatment of multidrug-resistant falciparum malaria. Antimicrob Agents Chemother 1998; 42: 135-39.

17 Ndayiragije A, Niyungeko D, Karenzo J, et al. Efficacy of therapeutic combinations with artemisinin derivatives in the treatment of non complicated malaria in Burundi. Trop Med Int Health 2004; 9: 673-79.

18 von Seidlein L, Bojang K, Jones P, et al. A randomized controlled trial of artemether/benflumetol, a new antimalarial and pyrimethamine/sulfadoxine in the treatment of uncomplicated falciparum malaria in African children. Am J Trop Med Hyg 1998; 58: 638-44

19 Hatz C, Abdulla S, Mull R, et al. Efficacy and safety of CGP 56697 (artemether and benflumetol) compared with chloroquine to treat acute falciparum malaria in Tanzanian children aged $1-5$ years. Trop Med Int Health 1998; 3: 498-504.

20 White NJ, van Vugt M, Ezzet F. Clinical pharmacokinetics and pharmacodynamics and pharmacodynamics of artemetherlumefantrine. Clin Pharmacokinet 1999; 37: 105-25.

21 Ezzet F, van Vugt M, Nosten F, Looareesuwan S, White NJ. Pharmacokinetics and pharmacodynamics of lumefantrine (benflumetol) in acute falciparum malaria. Antimicrob Agents Chemother 2000; 44: 697-704.

22 Ezzet F, Mull R, Karbwong J. Population pharmacokinetics and therapeutic response of G9P56697 (artemether+benflumetol) in malaria patients. Br J Clin Pharmacol 1998; 46: 553-61.

23 White NJ, Olliaro PL. Strategies for the prevention of antimalarial drug resistance: rationale for combination chemotherapy for malaria. Parasitol Today 1996; 12: 399-401.

24 Legros D, Johnson K, Houpikian P, et al. Clinical efficacy of chloroquine or sulfadoxine-pyrimethamine in children under five from south-western Uganda with uncomplicated falciparum malaria. Trans R Soc Trop Med Hyg 2002; 96: 199-201.

25 Priotto G, Kabakyenga J, Pinoges L, et al. Artesunate and sulfadoxine-pyrimethamine combinations for the treatment of uncomplicated Plasmodium falciparum malaria in Uganda: a randomized, double-blind, placebo-controlled trial. Trans R Soc Trop Med Hyg 2003; 97: 325-30.

26 Anon. Severe falciparum malaria: World Health Organization, communicable diseases cluster. Trans R Soc Trop Med Hyg 2000; 94 (suppl 1): S1-90.

27 Snounou G. Genotyping of Plasmodium spp: nested PCR. Methods Mol Med 2002; 72: 103-16.

28 Mansor SM, Navaratnam V, Yahaya N, Nair NK, Wernsdorfer WH, Degen PH. Determination of a new antimalarial drug, benflumetol, in blood plasma by high-performance liquid chromatography. J Chromatogr B Biomed Appl 1996; 682: 321-25.

29 Fogg C, Bajunirwe F, Piola $\mathrm{P}$, et al. Adherence to a 6-dose regimen of artemether-lumefantrine for treatment of uncomplicated Plasmodium falciparum malaria in Uganda. Am J Trop Med Hyg 2004; 71: 525-30.

30 Bakshi R, Hermeling-Fritz I, Gathmann I, Alteri E. An integrated assessment of the clinical safety of artemether-lumefantrine: a new oral fixed-dose combination antimalarial drug. Trans R Soc Trop Med Hyg 2000; 94: 419-24.

31 Brockman A, Price RN, van Vugt $M$, et al. Plasmodium falciparum antimalarial drug susceptibility on the north-western border of Thailand during five years of extensive use of artesunatemefloquine. Trans R Soc Trop Med Hyg 2000; 94: 537-44.

32 Stepniewska K, Taylor WR, Mayxay M, et al. In vivo assessment of drug efficacy against Plasmodium falciparum malaria: duration of follow-up. Antimicrob Agents Chemother 2004; 48: 4271-80.

33 Wernsdorfer WH. Coartemether (artemether and lumefantrine): an oral antimalarial drug. Expert Rev Anti Infect Ther 2004; 2 181-96.

34 Wernsdorfer WH, Landgraf B, Kilimali VA, Wernsdorfer G. Activity of benflumetol and its enantiomers in fresh isolates of Plasmodium falciparum from East Africa. Acta Trop 1998; 70: 9-15.

35 Williams HA, Jones CO. A critical review of behavioural issues related to malaria control in sub-Saharan Africa: what contributions have social scientists made? Soc Sci Med 2004; 59: 501-23. 